\section{Enzymatic Hydroxylations in Industrial Application}

\author{
Hans G. Kulla*
}

Abstract. In 1983, Lonza Ltd. initiated a small research group serving as a nucleus for the introduction of biotechnology to our organic intermediates and fine chemicals business. Over the past years we experienced vigorous growth and two projects reached industrial maturity: $i$ ) 6-Hydroxynicotinic acid (1) is a versatile building block derived by regiospecific hydroxylation of nicotinic acid (or niacin) (2), a B-group vitamin produced by Lonza. Thus, our first biotechnological product is fully integrated into the main product stream of Lonza's chemical production. ii) L-Carnitine (3) is synthesized by stereospecific hydroxylation of 4-butyrobetaine (4) in a $\beta$-oxidation process analogous to fatty-acid degradation. L-Carnitine (3) is a vitamin-like substance with interesting physiological capacities. Both processes are based on interrupted catabolic pathways of microbial origin. Production is on a multi-ton scale.

\section{Introduction}

The spectacular successes of modern biotechnology are mainly based on the advances in molecular biology. These techniques of DNA handling allows to synthesize, on an industrial scale, polypeptides mostly of mammalian origin in microorganisms like Escherichia coli. However, another branch of more traditional biotechnology is also in turbulent and promising development: biotransformation. The archetype of such an integration of chemistry and biotechnology is the Reichstein synthesis of vitamin C [1]. Ideally, chemistry supplies economic precursors and efficient product isolation, whilst the critical step is catalyzed by an enzyme or a multitude of enzymes, or sometimes by whole cells. Certainly, the arsenal of modern biotechnology is also revolutionizing this field, and even for the venerable and highly successful Reichstein process, alternative routes and shortcuts are being worked out employing cloned genes [2].

Here, two new industrial processes are presented briefly, one involving a regiospecific hydroxylation of a heteroaromatic ring, the other one comprising the stereospecific introduction of an $\mathrm{OH}$ group to an aliphatic C-atom.

\section{Production of 6-Hydroxynicotinate $(=6$ - Hydroxypyridine-3-carboxylate; 1)}

6-Hydroxynicotinic acid (1), a derivative of niacin (2) serves as a versatile building block chiefly in the synthesis of modern insecticides [3]. Chemical synthesis of 6substituted niacin is described in [4], but the

*Correspondence: Dr. H.G. Kulla

Table. Production of 6-Hydroxynicotinic Acid (1)

Phase 1:

Hydroxylation

Phase 2: add biomass air, $30^{\circ}, \mathrm{pH} 7$ centrifuge and dry

over all yield $\quad>90 \%$

purity

$>99 \%$
Chimia 45 (1991) 81-85

(C) Schweiz. Chemiker-Verband; ISSN 0009-4293

formation and subsequent separation of byproducts makes the cost prohibitively high. Niacin (or nicotinic acid) (2), a vitamin of the $\mathrm{B}_{2}$ group, is produced in large quantities in our chemical plant in Visp. Some years ago, in a sample of mother liquor from the production plant an unusual precipitate of white crystals was noticed. Analysis revealed that it was 6-hydroxynicotinate (1). As there was no purely chemical pathway known that could explain the formation of this derivative, the problem was handed over to our then very new biotechnology team. The predominant microorganism was isolated from the slightly turbid solution, and after identification we called it Achromobacter xylosoxidans LKl. As it turned out, this organism grows with niacin (2) as the sole source of energy, carbon and nitrogen and catabolises it through a well described pathway [5] (Scheme 1). Remarkably, at niacin (2) concentrations higher than $1 \%$ the second enzyme of the pathway, that is the 6 hydroxynicotinate hydroxylase (decarboxylating), gets strongly inhibited, whereas the niacin hydroxylase operates unaffected churning out the desired derivative.

CHIMIA $45(1991) \mathrm{NF} 3(\mathrm{MIT})$

Scheme 1. Degradation of Nicotinic Acid (2)

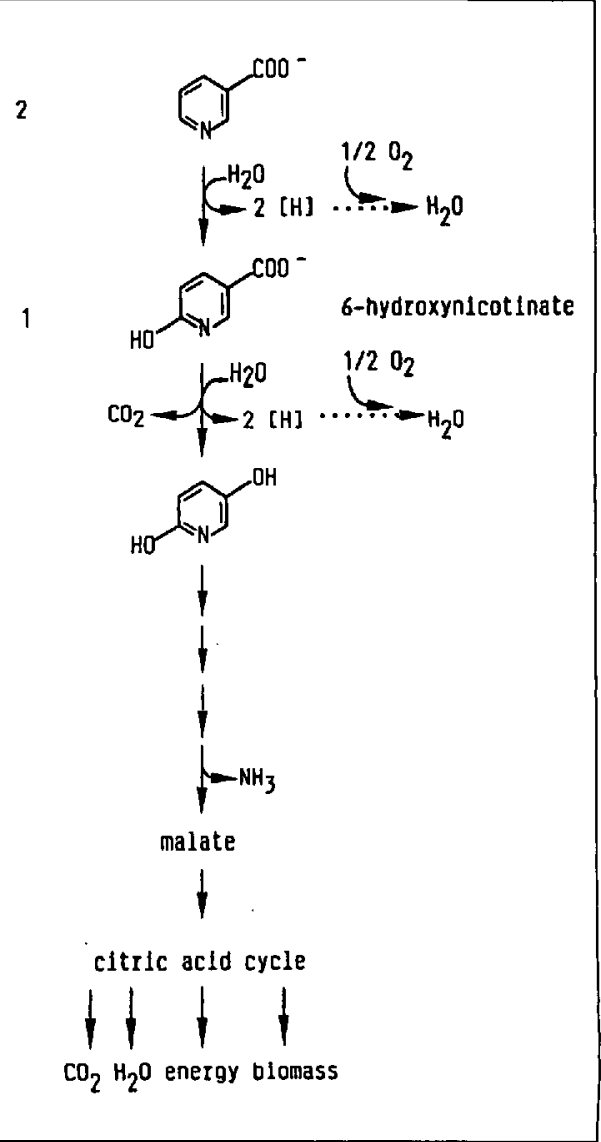

Apparently, in the mother-liquor mentioned above the niacin (2) concentration was at a threshold level allowing slow growth of the contaminating Achromobacter species, while the larger part of the substrate was channeled through the first enzymatic step only and excreted as 6-hydroxynicotinate (1).

This observation was translated into an industrial process [6]. To keep things simple but efficient, we run the process in a two stage mode as shown in the Table. As we are using basic, unit operations here like (continuous) fermentation, filtration, centrifugation, storing, and handling of active biomass, this process proved to be very effective for training our evolving piloting team. This team also demonstrated that

Production of biocatalys

grow cells in fermenter (chemostat) on niacin store biomass in cooled tank

$65 \mathrm{~g} / \mathrm{l}$ niacin (final concentration) in $\mathrm{H}_{2} \mathrm{O}$

incubate for $\sim 12 \mathrm{~h}$ (depending on oxygen transfer rate [27]) separate biomass (filtration or centrifugation) for recycling precipitate 6-hydroxynicotinic acid by addition of acid 


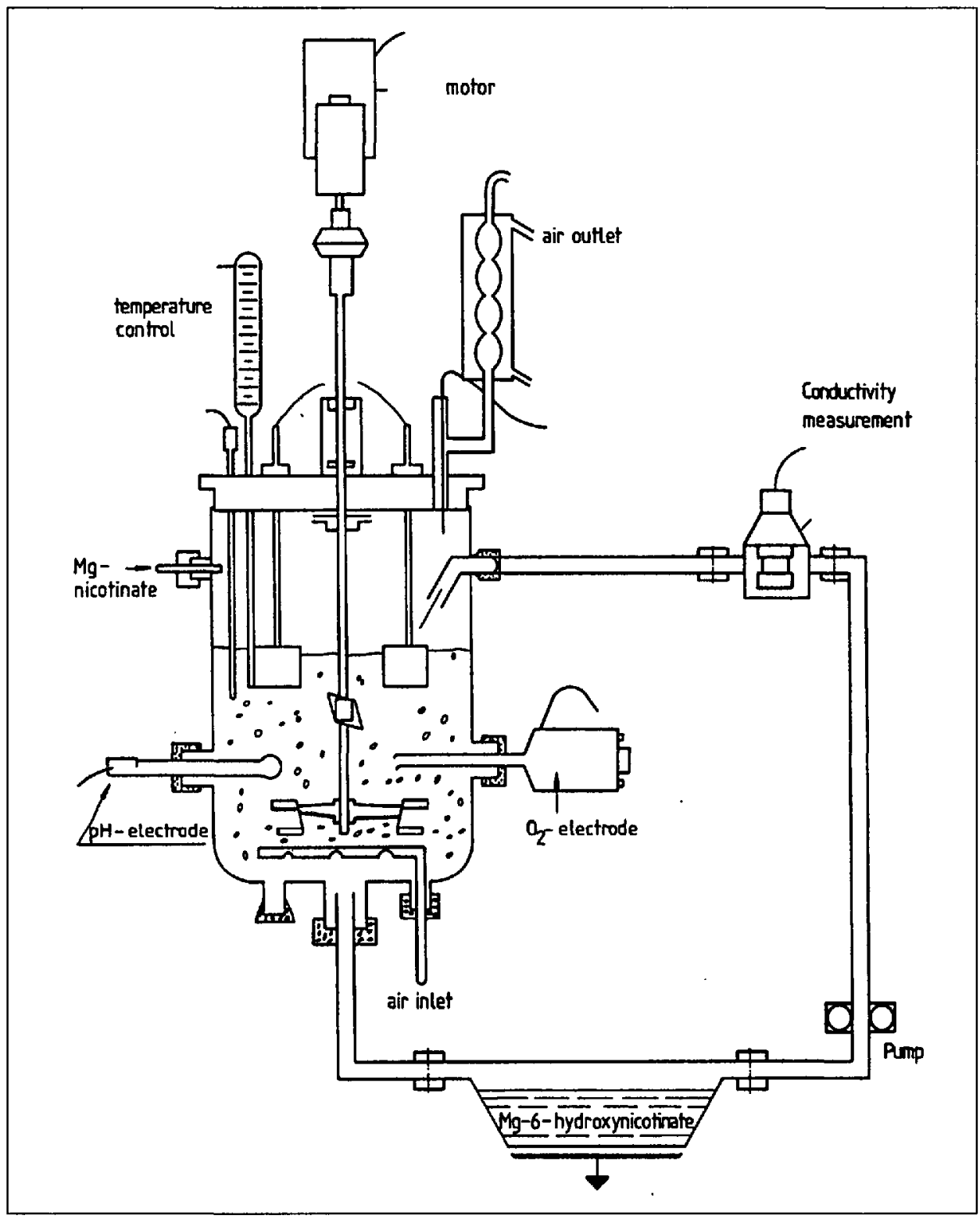

Fig. I. Continuous production of 6-hydroxynicotinate (1). The system is based an the observation that $\mathrm{Mg}$ nicotinate, the reactant, is soluble at neutral $\mathrm{pH}$ whereas the $\mathrm{Mg}$ salt of the product, that is 6-hydroxynicotinate (1), crystallizes out. See text for details.

Scheme 2. Anaerobic L-Carnitine (3) Respiration in Enterobacteria<smiles>C[N+](C)(C)C[C@H](O)CC(=O)[O-]</smiles>

3

L-camitine<smiles>C[C@H]1C[C@H](C)[C@@H](O)C1</smiles><smiles>C[N+](C)(C)C/C=C/C(=O)[O-]</smiles>

crotonobetaine

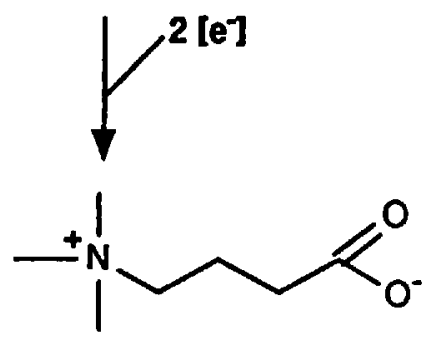

integration of the two phases into an efficient one pot fed-batch operation is possible. Here, a product-concentration of $74 \mathrm{~g} / \mathrm{l}$ can be reached in $25 \mathrm{~h}$.

To make things more intriguing, we also developed a continuous process (Fig. I) [7], which may be designated a 'pseudocrystal fermentation' according to Yamada's definition [8]. The interesting feature is that a solid reactant enters the fermenter, and the product is also harvested in a crystalline state. We take advantage of the fact that the $\mathrm{Mg}$ salt of niacin (2) is a 100 times more soluble in $\mathrm{H}_{2} \mathrm{O}$ at neutral $\mathrm{pH}$ than $\mathrm{Mg}-6$ hydroxynicotinate. Using conductivity measurement, the concentration of $\mathrm{Mg}$ nicotinate in the fermenter is regulated to $3 \%$ by direct addition of the salt. No buffers or nutrients are added, the $\mathrm{pH}$ is regulated to neutral by addition of nicotinic acid (2) or MgO. Active Achromobacter rylosoxidans LKI cells, grown beforehand in a fermenter on nicotinate (2), are added to a concentration of about $10^{10}$ cells $/ \mathrm{ml}$. Fortunately, the activity of the cells remains for a few days. The biotransformation yields 1 which precipitates as its $\mathrm{Mg}$ salt and is collected in a settler. The resulting drop in conductivity is compensated by activating the Mg-niacin pump. We managed to run the system in the laboratory for a few days despite technical problems stemming from plugging caused by product crystals. The productivity reached was ca. $1.5 \mathrm{~g} / \mathrm{l} / \mathrm{h}$. Obviously, such a system would need some clever engineering on a large scale.

So far, we are producing ton-lots of 6hydroxynicotinic acid (1) in the less ambitious two phase system described above. The product is then handed back to the chemists who transform it to valuable building blocks like 6-chloro- (5), or 6amino- (6), or 5,6-dichloronicotinic acid (7), 2,3,5-trichloropyridine (8), and, after reducing the $\mathrm{COOH}$ group, 6-chloro-3hydroxy-methylpyridine (9) [9]:

\section{Production of L-Carnitine (3)}

Having been successful with a catabolic pathway in the case described above, we followed a similar strategy for the production of L-carnitine (3) [10].

In the literature, there is a wide variety of procedures described for the chemical or biochemical preparation of $\mathrm{L}$-carnitine (3), (see e.g. [11]). The system based on the anaerobic L-carnitine (3) respiration of Escherichia coli and other Enterobacteria appears particularly interesting. In the absence of $\mathrm{O}_{2}$, these bacteria can use alternative respiratory electron acceptors like nitrate, nitrite, or chlorate, but also organic compounds like malate, which is reduced to succinate via fumarate [12]. In close analogy, L-carnitine ( 3 ) can be dehydrated to (trans-) crotonobetaine (10), which in turn serves as an anaerobic electron-acceptor yielding 4-butyrobetaine (4) as discovered 
by Kleber's group in Leipzig some years ago [13] (Scheme 2).

The reductive step can be inhibited by addition of nitrate, which is the preferred electron acceptor. In such a system, a halfhalf equilibrium of crotonobetaine (10) and L-carnitine (3) is reached [14]. Isolation of Lcarnitine (3) from such a mixture is rather tedious. Note that in this system the natural flow of metabolites has to be reversed for the production purpose. Maybe a system that swims with the metabolic stream would be more effective.

From a soil sample, a microorganism was isolated by elective culture that grows with 4-butyrobetaine (4), crotonobetaine (10), L-carnitine (3) (but not D-carnitine), and betaine (11) as sole carbon-, nitrogen-, and energy-sources under aerobic conditions. Taxonomically, strain HK4 is situated between Agrobacterium and Rhizobium. A new genus for this group has not been defined yet (Prof. Auling, Univ. Hannover, personal communication).

Early on, we showed that degradation of 4,10 , and 3 are under coordinate induction, indicating that they are metabolically closely linked [15]. It was very encouraging to observe that during growth on $\mathbf{1 0}$ small amounts of 3 were excreted transiently, presumably triggered by an overflow mechanism.

Using the mutant strain HK13, an efficient L-carnitine (3) producer described below, we showed by ${ }^{18} \mathrm{O}$-incorporation studies that the $\mathrm{O}$-atom in $\mathbf{3}$ is derived from $\mathrm{H}_{2} \mathrm{O}$ and not from $\mathrm{O}_{2}$. Further biochemical analysis in our laboratory revealed that the pathway is analogous (but not identical) to fatty-acid degradation (Scheme 3). Activity of the enzymes can be assayed in vitro, provided the necessary cofactors CoA, ATP, and FAD are included. The enzymes were enriched, but purification was hampered by their irksome instability. It seems that the synthetase and the hydrolyase build a complex which is easily separated from the 4-butyrobetainyl-CoA dehydrogenase. The thioesterase activity seems to be integrated into the complex. 4-Butyrobetainyl-CoA (12), the key-intermediate of the pathway, was isolated from in vitro assays by ionexchange chromatography and its structure confirmed by 'H-NMR.

As we were planning to use whole cells for the production, it seemed important to study the uptake and excretion of the betaines. The uptake is mediated by a specific and inducible periplasmic binding proteindependent mechanism [16]. These highly efficient transporters are known to use ATP in order to energize uptake even against a steep concentration gradient [17]. Interestingly, the excretion of the product $L$ carnitine (3) seems to be mediated through the same channel by a counterflux mechanism [18]: in the productive mutant described below the excretion of 3 is concomitant with the uptake of 4-butyrobetaine (4), reminiscent of a revolving door

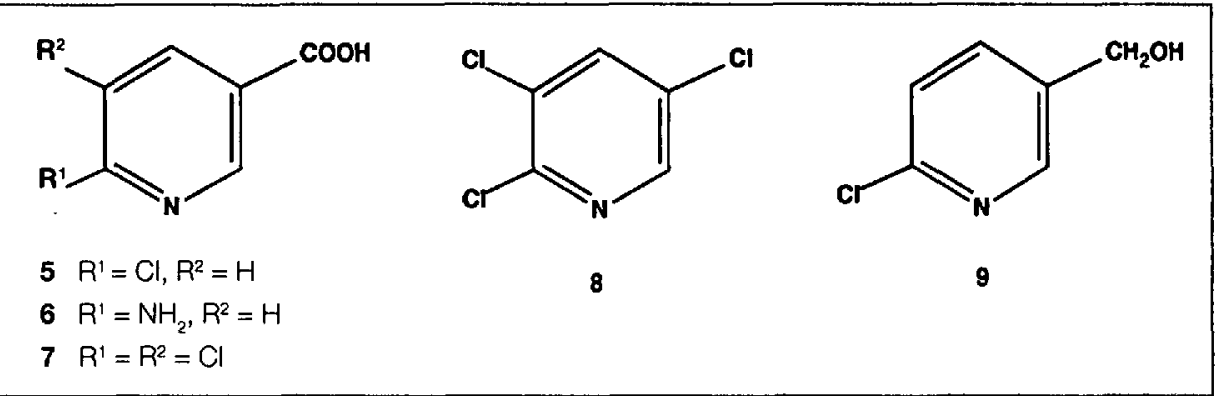

Scheme 3. Pathway for the Degradation of the Betaines in Strain HK 4 and $H K$ 13. The latter mutant has a blocked $\mathrm{L}$-carnitine dehydrogenase as indicated by the broken arrow. This strain excretes the accumulated l.-carnitine (3).

L-carnitine dehydrogenase as indicated by the broken arrow. This strain excretes the accumulated I.-cam

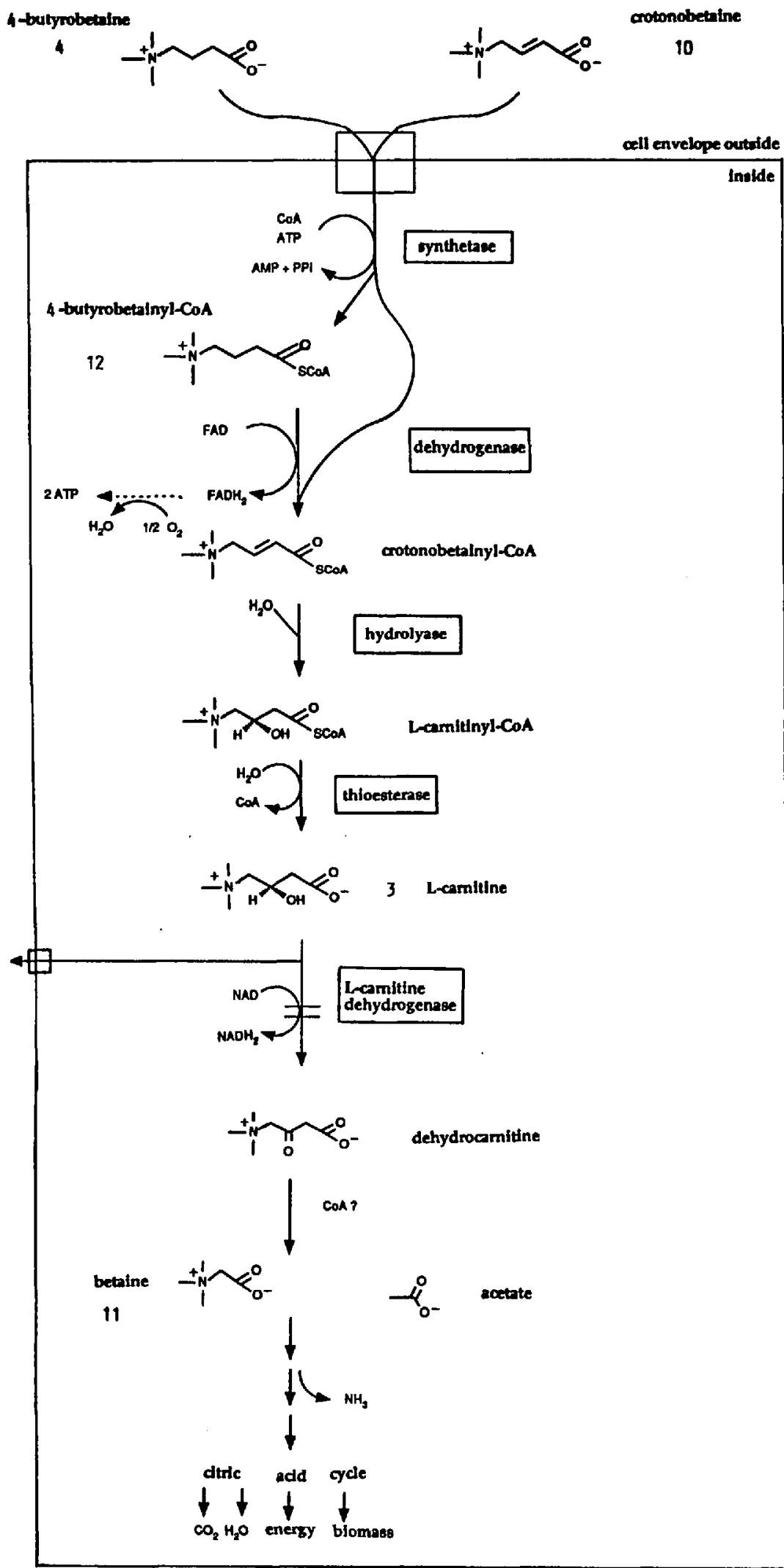


CHIMIA 45 (1991) Nr.3 (März)

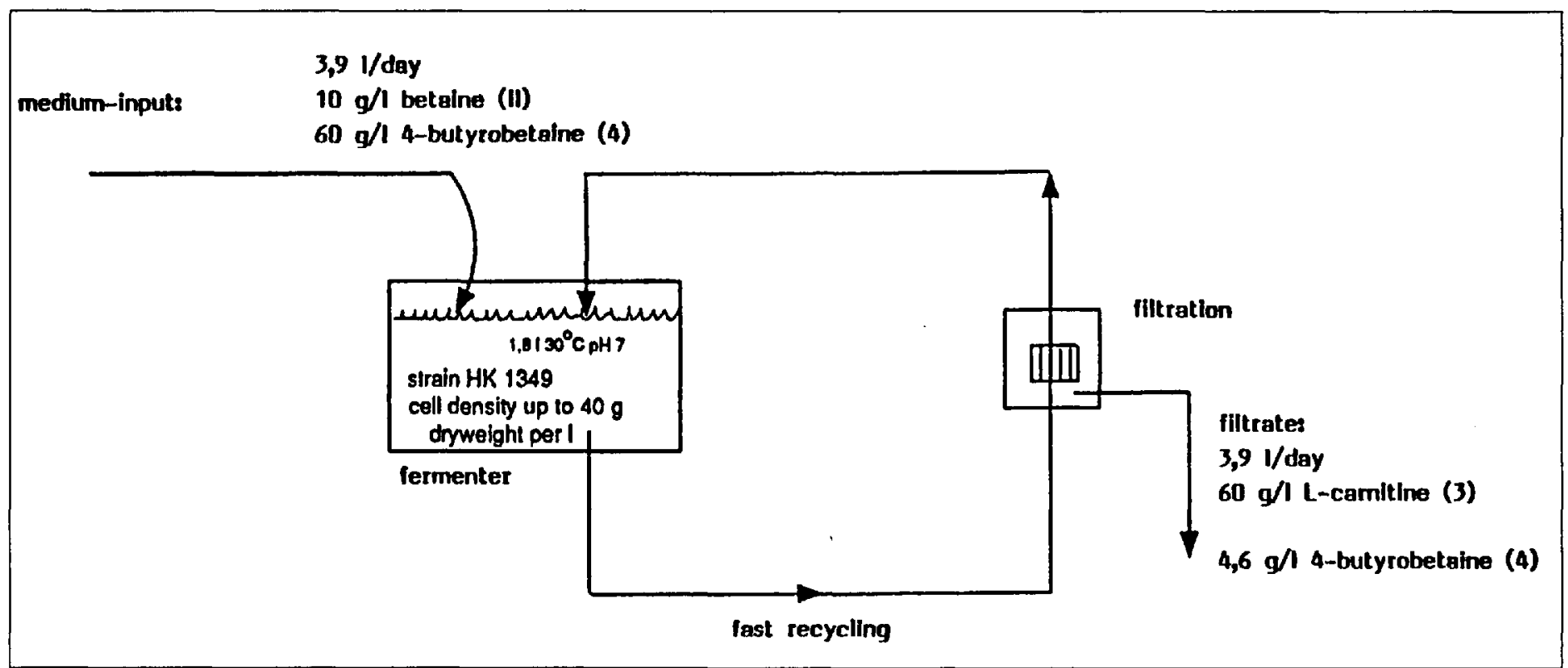

Fig. 2. Laboratory-scale continuous recycling culture for the production of L-carnitine (3)

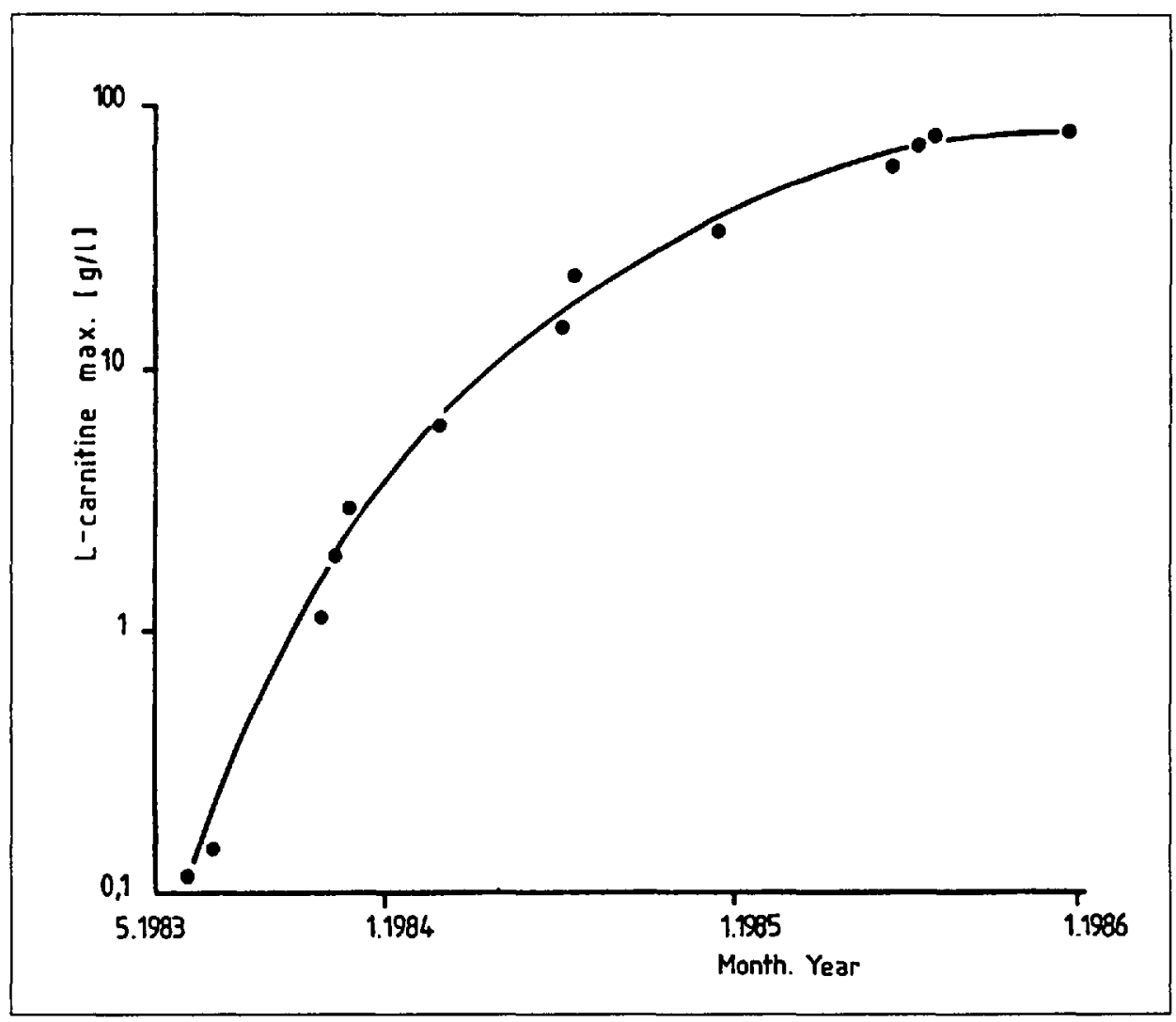

Fig. 3. Maximum L-carnitine (3) concentrations achievable as a function of our research and development efforts The project was initiated in the middle of 1983. Note the logarithmic scale.

mechanism. We were more than lucky to find a strain that provides an efficient excretion mechanism ex ovo.

L-Carnitine dehydrogenase (Scheme 3) was removed irreversibly from the wildtype HK4 by frameshift-mutagenesis followed by traditional counterselection, yielding strain HK13. This strain transforms 4butyrobetaine (4) and crotonobetaine (10) quantitatively into L-carnitine (3) and excretes it as described above. Needless to say that the enantiomeric excess of the product is $100 \%$. However, only growing cells are productive. This is probably due to the fact that the complex cofactor regeneration plus the transport are energy consuming. We believe that it is this investment of energy that drives the reaction to completion. Noeduct is left, which greatly facilitates isolation of the product. As a growth substrate, betaine (11) is best suited, maybe because it 'heals' the interrupted pathway (see Scheme 3). No complex nutrients need to be added.

HK13 suffers from product inhibition at L-carnitine (3) concentration higher than 30 $\mathrm{g} /$, but we have isolated resistant mutants that tolerate $70 \mathrm{~g} / 1$ and more. However, these mutants had lost their affinity to the educt 4 butyrobetaine (4), which became annoyingly apparent in the continuous production system described below. A strain development program yielded a compromise mutant with sufficient resistance towards high L-carnitine (3) concentrations and a tolerable uptake affinity towards the educt 4.

We designed a continuous production system with cell recycling as shown in Fig. 2 . The rationale behind this approach is twofold: Firstly, the resulting high concentration of biomass in the fermenter should lead to high biotransformation efficiency in a continuous mode. Secondly, it is known from the literature that cells in such an apparatus can go into a so-called maintenance state [19], which is characterized by high metabolic activity at very low growth rates. In our system, we calculated that the doubling time of cell-growth is in the range of 4 days, while the L-carnitine (3) production stays at a high level $(5.4 \mathrm{~g} / \mathrm{l} / \mathrm{h})$. Thus, the coupling between growth and production is largely annulated in our favor.

The activity proved to be absolutely stable. We were running the system for many weeks without detecting any loss in productivity. Obviously to maintain sterility and technical reliability of such a complex system with pumps, filters and a lot of pipework needs good engineering. In Visp, we piloted the process to a $400-1$ scale in a sophisticated fermentation installation, and together with a Japanese partner to a 2000-I scale [20].

Besides the engineering considerations mentioned, continuous operation has the intrinsic disadvantage that $100 \%$ turnover is not possible at high dilution rates. In our operation, $7-8 \%$ of the reactant leaves the fermenter unprocessed (see Fig . 2). For the low-affinity strains mentioned above this value can be as high as $20 \%$, prohibiting cost effective product isolation. To overcome this problem, the process was adapted to fedbatch operation[28]. Of course, volume productivity is ca. 5 times lower here, but tumover of reactant to product is better 
(99.5\%), which certainly facilitates purification.

How long did it take to develop the system starting from a soil sample to a labprocedure ready for scale up? To show development, we choose as a parameter the maximum L-carnitine (3) concentration that we were able to reach over the time scale in months and years (Fig. 3). It took roughly 2 $1 / 2$ years to push the concentration up over three orders of magnitude to make biotechnology competitive with our elegant inhouse chemical racemic resolution process [21]. It was startling to see the smooth development of the curve with no reflection at all of the breakthroughs and, more often, the débâcles which we experienced through these years.

Interestingly, eucaryotes like ourselves also synthesize L-carnitine (3) from 4butyrobetaine (4) which in turn is derived by a complicated route from L-lysine [22]. However, the reaction mechanism is completely different from the hydrolyase mechanism employed by our process, as the introduced oxygen is derived from $\mathrm{O}_{2}$ (Scheme 4).

In this unusual intermolecular dioxygenase mechanism, $\alpha$-ketoglutarate (13) as a cosubstrate accepts one of the two O-atoms and decarboxylates to succinate (14), whereas the other $\mathrm{O}$-atom gets integrated into 3 (for a recent paper, see [23]). There is one bacterial strain (Pseudomonas sp. AK1) described in the literature [24] that catabolises 4 through 3 by this mechanism. This metabolic type, which grows with 4 and 3 but not with crotonobetaine (10), was also isolated by us from soil, but we failed to turn it into a L-carnitine (3) producer, probably due to complications of $\alpha$-ketoglutarate (13) regeneration.

The rôle of L-carnitine (3) in eucaryotic metabolism is the transport of fatty acids across the membranes into the mitochondria. Thus, it has a key function in substrate flux and energy generation. Some clinical applications were discussed recently [25]. Another exciting application is the enhancement of uptake of drugs like antibiotics and also polypeptides through the nasal membrane by acylesters of L-carnitine [26].

\section{Epilogue}

It is Lonza's outspoken strategy to maintain and strengthen its position as the leading producer of fine chemicals. We demonstrated that integration of biotechnology into this concept contributes significantly to reach this goal.

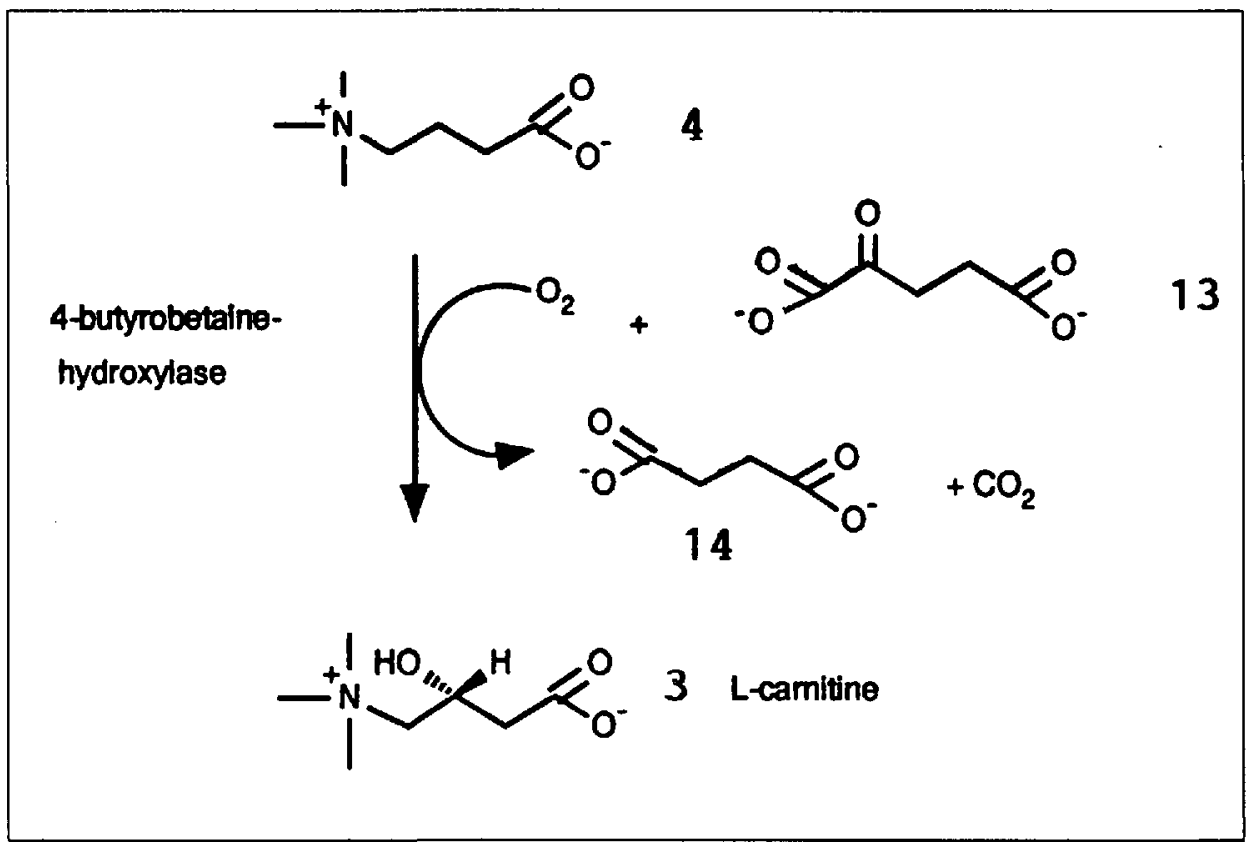

Scheme 4. Alternative Route from 4-Butyrobetaine (4) to L-Carnitine (3)

Received: December 3, 1990

[1] T. Reichstein, A. Grüssner, Helv. Chim. Acta 1943, 17, 311.

[2] J.Boudrant, Enzyme Microb.Technol.1990,12, 322.

[3] Eur. Pat. Appls. 0292822; $0302389,0302833$.

[4] D. Briancourt, J. Gilbert, Chim. Therap. 1973 2,226; D.Quarroz, to Lonza, Eur. Pat.0084118, 1986

[5] E.J. Behrman, R.Y. Stanier, J. Biol. Chem. 1957, 228, 923

[6] P. Lehky, H. Kulla, S. Mischler, to Lonza, Eur. Pat. Appl. 0152948, 1985.

[7] H. Kulla, P. Lehky, to Lonza, Eur. Pat. Appl 0152 949, 1985.

[8] T. Nagasawa, C.D. Mathew, J. Mauger, H. Yamada, Appl. Environ. Microbiol. 1988, 54, 1766.

[9] D. Quarroz, D. Worsch, U. Arni, Book of Abstracts of the 7th International Congress of Pesticide Chemistry - Hamburg (August 90), Eds. H. Frehse, E. Kessler-Schmitz, and S. Conway, IUPAC/GDCh, 1990, Vol. 1, p. 114

[10] H. Kulla, P. Lehky, to Lonza, Eur. Pat. Appl. 0158194, 1985; H.Kulla, P.Lehky, A.Squaratti, to Lonza, Eur. Pat. Appl. 0195944, 1986.

[11] R. Vocffray, J.-C. Perlberger, L. Tenud, J. Gosteli, Helv. Chim. Acta 1987, 70, 2058; B.-n. Zhou, A.S. Goplan, F. Van Middlesworth, W.R. Shieh, C.J. Sih, J.Am. Chem. Soc, 1983 105, 5925; J.-P. Vandecasteele, Appl. Environ. Microbiol. 1980, 39, 327; M.Kitamura, T.Ohkuma, O. Takaya, R. Noyori, Tetrahedron Lett. 1988, 29, 1555.

[12] J. Macy, H. Kulla, G. Gottschalk, J. Bacteriol. 1976, $125,423$.

[13] H. Seim, R. Ezold, H.-P. Kleber, E. Strack, Z Allg. Mikrobiol. 1980, 20, 591.

[14] H. Seim, H.-P. Kleber, Appl. Microbiol Biotechnol. 1988, 27, 538

[15] H.G. Schlegel, 'Allgemeine Mikrobiologie', Thieme Verlag, Stuttgart, 1985.
[16] S. Nobile, J. Deshusses, J. Bacteriol. 1986, I68, 780; S. Nobile, D. Baccino, T. Takagi, J. Deshusses, ibid. 1988, /70, 5236; S. Nobile, D. Baccino, J. Deshusses, FEBS Lett. 1988, 233 , 335; S. Nobile, J. Deshusses, Biochimie 1988, 70, 1411; S. Nobile, J. Deshusses, J. Chromatogr. $1988,449,331$.

[17] G.F.-L. Ames, A.K. Joshi, J. Bacteriol. 1990 172,4133 .

[18] S. Nobile, Thesis No. 2282, Université de Geneve, 1987.

[19] H.W. van Verseveld, M. Arbige, W.R. Chesbro, Trends Biotechnol. 1984, 2, 8 .

[20] F.W.J.M.M. Hoeks, H. Kulla, H.-P. Meyer submitted to J. Biolechnol.

[21] L. Tenud, J. Gosteli, to Lonza, Eur. Pat. 157315 , 1984.

[22] W.K. Paik, S. Nochumson, S. Kim, Trenels Biochem. Sci. 1977, July, 159.

[23] R.C. Petter, S. Banerjee, S. England, J. Org Chem. 1990, 55, 3088.

[24] G. Lindstedt, S. Lindstedt, I. Nordin, Biachemistry 1977, 16, 2181

[25] Lancet 1990, 335, 631, 981, 1215.

[26] J.Alexander, A.J. Repta, J.A. Fix, to Merck, Eur. Pat. Appl. 0215697, 1987; J. Alexander, A.J. Fix, to Merck, Eur. Pat. Appl.0162764, 1985; S. Kagaya, N. Inaba, T. Sonobe, to Yamanouchi Pharmaceutical Co., Jap. Pat. Appl. 01308235 1989.

[27] F.W.J.M.M. Hoeks, H.-P. Meyer, D. Quarroz, M. Helwig, P. Lehky, in 'Opportunities in Biotransformation', Eds. L.G. Copping, R.E. Martin, J.A. Pickett, C. Bucke, and A.W. Bunch, Elsevier, London, 1990, p. 67-71.

[28] F.W.J.M.M. Hoeks, to Lonza, Eur. Pat. Appl. 0410430,1991 